\title{
(Kein) Widerspruch im Engagement
}

\section{Beobachtungen zum kritischen Potential bürgerschaftlich Engagierter in Wohlfahrtsverbänden}

\author{
Andreas Kewes $\cdot$ Chantal Munsch
}

Online publiziert: 29. Juni 2018

(C) Der/die Autor(en) 2018

Zusammenfassung Anhand von narrativen Interviews mit ehemaligen Engagierten wird bürgerschaftliches Engagement in Wohlfahrtsverbänden als ein Ort der Politisierung rekonstruiert. Der Text formuliert dabei folgende Beobachtung: Engagierte stellen sich nicht als per se kritische Subjekte dar. Vielmehr schildern sie, wie sie im Verlauf ihres Engagements in der Wohlfahrt starke Erfahrungen gemacht hätten, anhand derer sie eigene Werthaltungen entwickelten. Gleichzeitig hätten sie das, was sie im Engagementfeld beobachten, als konträr zu diesen Werthaltungen erlebt. Die Entwicklung einer solchen Werthaltung, mit der sich die Engagierten in Gegensatz zum Engagementfeld positionieren, analysieren wir als Widerspruch. Der Text rekonstruiert diesen Widerspruch sowohl hinsichtlich seiner Genese als auch hinsichtlich seiner Bestandteile sowie seines Bezuges zum Kontext der Wohlfahrt. Die These lautet, dass der Widerspruch insbesondere das widersprechende Subjekt verändert, weniger jedoch die Organisationsroutinen, die es adressiert. Theoretisch gerahmt werden die Befunde durch Hans Joas Idee von der Entwicklung von Wertbindungen als Folge von starken Erfahrungen sowie durch die Positionierungsanalyse von Gabriele Lucius-Hoene, Arnulf Deppermann und Rom Harré. Der Aufsatz leistet einen Beitrag zur Zivilgesellschaftsdebatte, insbesondere zu Überlegungen,

\footnotetext{
Der Aufsatz und die hierin verwandten Daten basieren auf der von der DFG geförderten Studie Menschen mit Migrationshintergrund in deutschen Wohlfahrtsverbänden. "Die Autor*innen danken der DFG für die finanziellen Mittel sowie Anne-Marie Stumpf, Moritz Müller und Naima Marie Brüggenthies für ihre hervorragende Unterstützung bei der Forschung und Publikationsvorbereitung.

Dipl.-Pol. A. Kewes · Prof. Dr. C. Munsch $(\varangle)$

Lehrstuhl für Erziehungswissenschaft mit dem Schwerpunkt Sozialpädagogik,

Fakultät II, Erziehungswissenschaft - Psychologie, Universität Siegen,

Adolf-Reichwein-Straße 2a, 57068 Siegen, Deutschland

E-Mail: Chantal.munsch@uni-siegen.de

Dipl.-Pol. A. Kewes

E-Mail: andreas.kewes@uni-siegen.de
} „Abgebrochene Zugänge zu bürgerschaftlichem Engagement. Eine qualitative Studie am Beispiel von 
ob und wie bürgerschaftliches Engagement gestalterisch in Gesellschaft eingreifen kann.

Schlüsselwörter Bürgerschaftliches Engagement · Emotion · Qualitative Sozialforschung $\cdot$ Werte $\cdot$ Widerspruch

\title{
(No) voice in volunteering
}

Observations on the critical potential of civic volunteers at charitable associations

\begin{abstract}
Using narrative interviews with ex-volunteers, civic engagement at charitable associations is reconstructed as a space for politicisation. The text formulates the following observation: volunteers do not present themselves as critical subjects per se; instead, they describe how they have powerful experiences during the course of their volunteering for the charitable association which lead them to develop their own values. At the same time, they recount how they experience what they observe in the field of volunteering as being contrary to those values. We analyse the contradictory nature of the development of these values, through which the volunteers position themselves as in opposition to the field of volunteering. The text reconstructs that contradiction in terms not only of its genesis but also of the elements it comprises and how it links in with the charitable context. Our theory is that the contradiction particularly changes the subject involved in contradicting, and less so the organisational routines at which it is addressed. The basis of this analysis comes from Hans Joas's idea of value commitments developing as a consequence of powerful experiences, plus the positioning theory by authors such as Gabriele LuciusHoene, Arnulf Deppermann and Rom Harré. This essay plays a part in the debate on civil society, and especially on thoughts about whether and how civic engagement can intervene and shape society.
\end{abstract}

Keywords Emotion - Qualitative Research · Values · Voice · Volunteering

Zahlreiche Studien zum bürgerschaftlichen Engagement betonen, wie bedeutsam dieses nicht nur politisch, sondern auch ökonomisch für moderne Sozialsysteme ist (Simonson et al. 2017, S. $31 \mathrm{f}$; Dunn et al. 2016). Mit dem Begriff der Politik ist hier - etwas vereinfacht - eine mitgestaltende und v. a. auch Veränderungen in Organisationen bewirkende Tätigkeit gemeint, während der Verweis auf die ökonomische Bedeutsamkeit das Politische eher ausklammert und die Verwertbarkeit des Engagements in den Vordergrund stellt. So betrachtet wird ein latenter Widerspruch deutlich zwischen dem Eigensinn bürgerschaftlichen Engagements als Ausdruck von Öffentlichkeit, Zivilität und Demokratie (Evers 2010, S. 283; Roth 2010, S. 617 ff.) einerseits und seinem Wert als ökonomische Ressource andererseits (vgl. auch Dahme und Wohlfahrt 2007). In diesem Kontext kommt der Erforschung des Abbruchs bürgerschaftlichen Engagements eine doppelte Rolle zu, ist damit doch sowohl das Verschwinden einer ökonomischen Ressource als auch das Verschwinden kritischer Einflussnahme verbunden. 
Es gibt in den letzten Jahren zunehmend Studien, die sich der zeitlichen Befristung und dem Abbruch bürgerschaftlichen Engagements widmen (Hustinx 2010; Rochester et al. 2010, S. 133 ff.). Diese Studien fokussieren allerdings zumeist eingegrenzte Phänomene, die relativ eindeutig entweder der Mikro-, Meso-, oder Makroebene zugeordnet werden können. Erklärt werden Abbrüche hier z. B. durch unklare Rollenanforderungen an Engagierte und nicht eindeutige Aufgabenbeschreibungen (Allen und Mueller 2013). Weiterhin werden Organisationsaspekte herausgestellt, wie etwa das Vorhandensein einer Kommunikation zwischen haupt- und ehrenamtlichen Kräften (Garner und Garner 2011). Auch individuelle Erwartungen, die nicht zu denjenigen der Organisation oder zu den Aufgaben passen, sowie abträgliche Dispositionen der Engagierten wie mangelnde Zeitressourcen oder (Un-)Zufriedenheit werden zur Erklärung herangezogen (Wilson 2012; Ehrhardt 2011). Zuletzt gibt es Versuche, für die Erklärung von Abbruch individuelle, organisationsbezogene und makrosoziale Ebenen in der Analyse schlicht zu addieren, ohne sie aber aufzulösen oder ihre Interdependenz herauszuarbeiten (Ripamonti et al. 2017). ${ }^{1}$ Bei all diesen Erklärungsansätzen ist auffallend, dass sie sich nur auf bestimmte Ursachen beschränken und dabei weitgehend auf der Ebene der Engagementförderung verbleiben. Dabei bleibt der Zusammenhang zwischen der individuellen Motivation und den strukturellen Bedingungen, in denen das Engagement stattfindet, weitgehend ausgeblendet. Die Engagierten kommen v. a. als pädagogisch zu motivierende und institutionell über passend zugeschnittene Aufgaben zu organisierende in den Blick - aber nicht mehr als politische Akteur*innen, die gestalten und verändern wollen. ${ }^{2}$

Die im Folgenden vorgestellten Ergebnisse aus einer Interviewstudie zeigen, dass sich ein Verständnis der Beendigung bürgerschaftlichen Engagements, welches sich auf einzelne Maßnahmen des Managements von Engagement oder auf die individuelle Motivation (und damit auf nur eine sozialwissenschaftliche Ebene) beschränkt, nicht aufrechterhalten lässt. Es lassen sich auch im vermeintlich systemunterstützenden und deswegen oft v. a. ökonomisch betrachteten wohlfahrtsstaatlichen Engagement politische (bzw. politisierende) Momente finden, welche die Ebene der individuellen Motivation mit derjenigen der strukturellen Bedingungen der Wohlfahrtsverbände verbindet. Dies verweist auf die Notwendigkeit einer Analyseperspektive, welche das engagierte Subjekt stärker mit dem institutionellen Kontext zusammen denkt. Entsprechend wird in dem vorliegenden Aufsatz die Unzufriedenheit von ehemaligen Engagierten mit ihren Aufgaben, ihrer Organisation und ihrem Umfeld zum Gegenstand der Analyse gemacht. Der Text argumentiert entsprechend nicht aus systemischer Perspektive auf Zivilgesellschaften, sondern versucht, die Sichtweise der Engagierten zu rekonstruieren. Ziel ist es dabei, zu zeigen, wie wiederum über die Interpretation von Erfahrungen und die Artikulation von Widerspruch Erwartungen

\footnotetext{
${ }^{1}$ Bereits recht früh hat Albert O. Hirschman (1984) die Beendigung politischen Engagements als Aspekt von Karrieren beschrieben, die in ihrem Verlauf eher längeren Wellen der Dialektik von Privatwohl und Gemeinwohl folgten. Zwar liegt auch damit kein Verständnis von Engagementabbrüchen vor, mit dem wir arbeiten wollen, aber Hirschman (1984) koppelt auf diese Weise unterschiedliche sozialwissenschaftliche Ebenen (gesellschaftliche Trends mit individuellen Biografien) auf interessante Art und Weise.

2 Vgl. zu dieser Kritik auch van Dyk und Miesebach (2016).
} 
an Tätigkeiten und Organisationen entstehen bzw. sich verändern. Dadurch wollen wir eine Ergänzung des oben beschriebenen Forschungsstandes vorschlagen.

Für die dieser Arbeit zugrundeliegende Interviewstudie wurden ehemalige Engagierte aus Wohlfahrtsverbänden befragt. Ein Teil der Befragten erzählt das Engagementende als notwendig gewordene biografische Veränderung, etwa als Ausdruck eines sich wandelnden Zeitmanagements oder ausgelaufener Projekte. Ein anderer Teil der befragten Engagierten erzählt sich als ein kritisches Subjekt, das mit einer bestimmten Art und Weise des Umgangs mit Klient*innen oder Engagierten nicht einverstanden ist. ${ }^{3}$ Die letztgenannte Gruppe zog unser Interesse auf sich, weil ihre Art der dissidenten Sichtweise auf Engagement bislang noch kaum erforscht wurde. ${ }^{4}$ Diese Engagierten stellen ihren Engagementverlauf nicht als einen dar, der selbstverständlich oder zwangsläufig auf eine bestimmte Weise ablief, sondern sie erzählen, dass sie hinterfragt hätten, was in ihrem Engagement vor sich gegangen sei. Sie erzählen sich als durchaus kreativ im Umgang mit den ihnen gestellten Aufgaben. Die Engagierten setzen in den Interviews bestimmte Werte und Überzeugungen relevant und kritisieren, dass Handlungsweisen in den jeweiligen Engagementzusammenhängen diesen Werten nicht entsprächen. Damit positionieren sie sich in Gegensatz zu dem, was sie im Engagementfeld erleben. Wir analysieren im Folgenden diese Entwicklung einer eigenen Werthaltung, die in Gegensatz zum Engagementfeld gestellt wird. Diese Positionsentwicklung nennen wir Widerspruch ${ }^{5}$ und interessieren uns dabei insbesondere für deren Bestandteile.

Wir rekonstruieren bürgerschaftliches Engagement in Wohlfahrtsverbänden als einen Handlungskontext, der Wissen produziert über die Kritikwürdigkeit gesellschaftlicher Zustände und die Schwierigkeit, als notwendig erscheinende Veränderungen anzusprechen und durchzusetzen. Um die Entstehung und Artikulation dieses Engagiertenwissens genauer darzustellen und damit auch die Ambivalenzen einer Politisierung auszuleuchten, sind Überlegungen zur Entwicklung des Widerspruchs bzw. der Moralität der Engagierten maßgeblich. Diese reflektieren wir zunächst empirisch (Kap. 2). Wir rekonstruieren die Genese eines Fallbeispiels, anhand dessen wir zentrale Bestandteile des Phänomens Widerspruch erarbeiten. Die anschließende theoretische Abstraktion erläutert die Idee, dass es sich bei solchen Widerspruchsakten immer auch um Positionierungen handelt, welche die eigene Position zu derjenigen der Organisationen im sozialen Feld in Relation setzt und ggf. neu anordnet (Kapitel drei). Ziel dieses Artikels ist eine differenzierte Rekonstruktion eines Phä-

\footnotetext{
3 Insofern wir in diesem Artikel die Kritiken der Akteur*innen zum Gegenstand der Analyse machen, orientiert sich unsere Datenrekonstruktion sehr stark an der Perspektive der Soziologie der Kritik (z.B. Boltanski 2010; Boltanski und Thévenot 2007).

${ }^{4}$ Gegenwärtig wird unter dem Begriff rebellisches Engagement ein Diskurs über Engagement für Geflüchtete geführt. Dabei kommt den widerspenstigen und kritischen Aspekten des Engagements mehr Aufmerksamkeit zu. Die Kritik richtet sich aber zumeist gegen das europäische Asylregime und weniger beispielsweise gegen die Wohlfahrtsverbände, die vor Ort für die Betreuung zuständig sind (vgl. Graf 2016; van Dyk et al. 2016). Es geht bei den genannten Autorinnen darum, den Druck des Engagements auf die Politik nachzuzeichnen.

5 Damit nutzen wir in dieser Arbeit ein Verständnis von Widerspruch wie es beispielsweise bei Albert O. Hirschman (1974) zu finden gewesen wäre, also als Substantivierung des Verbs ,widersprechen“. Dies ist eine andere Begriffsverwendung als beispielsweise diejenige der dialektischen Philosophie wie sie etwa in der Marx'schen Theorie gebraucht wird (Widerspruch zwischen Kapital und Arbeit).
} 
nomens der Auseinandersetzung von Engagierten in wohlfahrtsstaatlichen Kontexten mit den dortigen Organisationen. Dies soll zum einen zu einem besseren Verständnis von bürgerschaftlichem Engagement aus der Perspektive von Engagierten beitragen. Zum anderen soll der Beitrag anregen, Prozesse der Engagementbeendigung differenzierter weiterzudenken.

\section{Datenerhebung und Methodik}

Das Forschungsprojekt, aus dem die hier vorgestellten Ergebnisse stammen, fragt nach Zugangs- und Abbruchsprozessen zu bürgerschaftlichem Engagement im Wohlfahrtsbereich. Die Ausgangsfragestellung lautete: Welcher soziale Sinn liegt der Beendigung eines bürgerschaftlichen Engagements zugrunde? ${ }^{6}$ Hierzu führten wir 18 narrative Interviews mit Engagierten, die ein unentgeltliches Engagement in einem Arbeitskontext der großen deutschen Wohlfahrtsverbände beendet haben. Für den vorliegenden Aufsatz wurden sieben Fälle intensiver ausgewertet. Dieses zweite Sampling begründet sich mit der spezifischen Passung der Interviews: Diese Engagierten stellen sich mit ihren Erzählungen als Widersprechende in einen Gegensatz zum Engagementfeld. Die nachfolgenden Ausführungen stellen einen Teil der Ergebnisse unserer Studie dar.

Den Ausgangspunkt der erhobenen Interviews bildet unsere Bitte, die Geschichte zu erzählen von dem Beginn der Überlegung, sich wohlfahrtsstaatlich zu engagieren, über das Engagement selbst bis hin zum Engagementende. Narrative Interviews bieten den ehemaligen Engagierten die Möglichkeit, eigenständig Erzählungen über eine gesamte Engagementbiographie zu entwickeln und dabei die Sequenzen Davor, Währenddessen und Danach sinnhaft einzuweben. Damit erhielten wir Erzählungen über den Engagementverlauf als einen Prozess (Kewes 2017; Snyder und Omoto 2008; Haski-Leventhal und Bargal 2008). In diesem Zusammenhang können die Befragten auch der Konstruktion des eigenen Selbst genügend Raum geben: Die eigene Veränderung - etwa als engagierte Person oder der Übergang von der engagierten zur hier nicht mehr engagierten Person - wird sowohl im Zeitverlauf erzählt als auch in seiner sozialen und moralischen Tragweite. Die Interviews dauerten zwischen 40 Minuten und drei Stunden und wurden vollständig transkribiert.

Den Feldzugang fanden wir maßgeblich über Multiplikator*innen bei den Wohlfahrtsverbänden sowie in kommunalen Engagementförderstellen. Im Sample sind verschiedene Altersgruppen, Geschlechter und Migrationserfahrungen sowie Wohlfahrtsverbände vorhanden. Die Auswertung erfolgte nach dem hermeneutischen Verfahren der Dokumentarischen Methode (vgl. Nohl 2017). Mit dieser Methode wird

\footnotetext{
${ }^{6}$ Ursprünglich fragten wir nach der Bedeutung eines Migrationshintergrundes für die Beendigung der Engagementverhältnisse in den Wohlfahrtsverbänden als Organisationen der Mehrheitsgesellschaft. Das Sample für die Interviews umfasste deswegen zunächst nur Engagierte mit einem Migrationshintergrund. Im Verlauf der Datenanalyse, welche - wie in rekonstruktiven Forschungsvorhaben üblich - den Prozess der Datenerhebung begleitet und diesen auch steuert (vgl. Glaser und Strauss 2005, S. 53-83), wurde jedoch deutlich, dass Migration für die Erzählungen über den Engagementverlauf, über das Bindende und das Abstoßende und somit für die Beendigung des Engagements keine Rolle spielt. Daraufhin wurde ein Migrationshintergrund bei der Suche nach Interviewpartner*innen nicht mehr vorausgesetzt.
} 
nach den geteilten Erfahrungen, die den Schilderungen zu Grunde liegen, gesucht sowie danach, wie über das Engagementhandeln geredet wird, das heißt, nach dem Orientierungsrahmen der Erzählungen. Die einzelnen Interviews wurden jeweils einzeln in Bezug auf die verhandelten Themen und Darstellungsweisen analysiert. Entsprechend der Methode wurden für eine tiefere Auswertung solche Passagen ausgewählt, die thematisch passend sind und die über eine hohe darstellerische Dichte verfügen (vgl. Nohl 2017, S. 30). Diese wurden sowohl fallintern als auch fallübergreifend systematisch verglichen, um dadurch auf kollektiv geteilte Wissensbestände schließen zu können. ${ }^{7}$ Die Interpretation der Daten erfolgte in der vierköpfigen Forschungsgruppe, zudem halbjährlich in Forschungswerkstätten; Zwischenergebnisse wurden auf Workshops und Konferenzen diskutiert. Der nachfolgende Text analysiert primär anhand eines exemplarischen Interviews ein von uns gefundenes gemeinsames Wissen der Engagierten, welches wir Widerspruch nennen.

\section{Empirische Analysen}

\subsection{Die Entwicklung von Widerspruch anhand konkreter Erfahrungen: ein Fallbeispiel}

Ganz allgemein haben wir festgestellt, dass die Interviewten in den Interviews einleitend ausführten, dass sie zu Beginn ihres Engagements noch keine rechte Vorstellung von diesem gehabt hätten. Vielmehr führen sie recht abstrakte ethische Begründung an, etwa Reziprozität. Diese Begründungen sind eher unspezifisch, es handelt sich um Ideen von Reziprozität oder Solidarität. Es wird einleitend kaum eine Idee von Widerspruch an einer konkreten Organisationspraxis artikuliert. Die Engagierten erzählen, wie sie erst im Verlauf ihres Engagements ganz konkrete Aufgaben gesucht und gefunden haben. Sie erzählen von bestimmten Beziehungen, und zwar solchen, von denen sie vorher nichts ahnten. Im Zusammenhang mit den sich erst im Engagementverlauf konkretisierenden Aufgaben und Beziehungen hätten sie dann auch zu ihrem spezifischen Standpunkt im Engagement gefunden. Insofern stellen sich die Engagierten nicht als per se kritische Subjekte dar, welche gewisse Strukturen schon immer verändern wollten, sondern es gilt, die Entwicklung von Widerspruch für den Engagementverlauf zu rekonstruieren.

Anhand der Rekonstruktion einer Falldarstellung wird im Folgenden exemplarisch nachgezeichnet, wie Engagierte in ihren Erzählungen alternative Sichtweisen auf Engagementroutinen entwickeln. Die Engagierte Linda beschreibt ihr Engagementerleben in zwei Altenstiften zunächst als besonders erfüllend und gewinnbringend, dann aber zunehmend auch als verstörend und verärgernd ${ }^{8}$ Eine Passage ihrer

\footnotetext{
7 Wissen ist für die nachfolgende Analyse eine zentrale methodologische Begrifflichkeit. Sie ist der Oberbegriff für all die Interpretationen, Schemata und Konstruktionen, welche soziales Handeln (zum Teil implizit) vorstrukturieren (vgl. Bohnsack 2010, S. $20 \mathrm{ff}$.). Methodologisch trachten wir in diesem Aufsatz danach, dieses Wissen zu rekonstruieren. Der Text sollte allerdings auch mit Gewinn lesbar sein, wenn keine Kenntnis der wissenssoziologischen Terminologie und Fundierung des Auswertungsverfahrens besteht.

8 Sämtliche Namen in diesem Aufsatz sind anonymisiert.
} 
Erzählung bekommt dabei eine besondere Bedeutung. Sie schildert hier nicht nur mit besonderer Emphase und sehr ausführlich, sondern benutzt hier ein bestimmtes Erlebnis, um exemplarisch darzustellen, wie unterschiedlich ihre eigene Haltung in der Pflege zu derjenigen der hauptamtlichen Kräfte sei. Sie beginnt diese Erzählpassage mit den Worten ,ich hatte einen ganz schlimmen Vorfall“ - und nimmt damit schon ihre Bewertung vorweg. Dann führt sie weiter aus:

„Und dann hatt' ich die eine Frau, die hab' ich mit nach draußen genommen, und die hatte dann 'n Rollstuhl, mir ist das in dem Moment auch nicht aufgefallen, da waren keine Fußstützen dran. Und normal hat 'n Rollstuhl Fußstützen. Dann bin ich mit der rumgefahren, die fand das auch schön, aber sie hatte dann auf einmal, als wenn sie Schmerzen hatte. Aber das war mir ja irgendwie klar, weil, die hatte die Fußstützen nicht, und dann denk' ich, ja, denk' ich: (überrascht) ,Die hat die Beine ja die ganze Zeit von sich aus hochhalten müssen. ' [...] Und ähm, die hatte danach solche Schmerzen ..."

An dieser Stelle des Interviews erzählt Linda die Beobachtung, dass ihr die Schmerzen der alten Dame nahegegangen seien. Hier zeichnet sich zunächst ein Miterleben mit der alten Frau, aber insbesondere durch den Tonfall der Erzählung auch schon eine gewisse Empörung ab. Diese wird im weiteren Verlauf der Schilderung noch deutlicher, wie anhand der folgenden Passage aus der Erzählung analysiert werden kann. Hier schildert Linda, wie sie zur zuständigen Ordensschwester gegangen sei, um auf diesen Sachverhalt aufmerksam zu machen:

„... da bin ich zu der Schwester gegangen. Ich sage: ,Hören Sie mal, so und so', [...], und das wurd' dann irgendwie abgetan äh, ach, als wenn sie so ((zögern)) ähm Aufmerksamkeit erzielen wollte. Und äh, da sag' ich, ich sag': ,Ja, die von Dingens hat doch/da/“ Ich sag': ,In dem Rollstuhl/“ Ich sag': (entrüstet) ,Die hat doch gar keine Fußstützen. Warum sind denn die Fußstützen da vorne“ „Ja, die müssen dann im Zimmer liegen.' Ja, gucken gegangen, Fußstützen nirgendwo zu finden, $[\ldots]^{\text {"10 }}$

Linda erzählt dieses Gespräch mit der Ordensschwester im Interview sehr lebendig nach. Damit verleiht sie dieser Interaktion eine zentrale Bedeutung für ihre Geschichte. Sie inszeniert sich dabei in einer bestimmten Weise: Als diejenige, die die Klientin tatsächlich ernst nehme, die schwierige Umstände angemahnt (,Hören Sie mal") und dabei zu Recht Empörung gezeigt habe. In dieser Empörung habe sie jedoch keine Anerkennung erfahren, ganz im Gegenteil: Sie sei mit ihrer Bewertung der Situation ,,irgendwie abgetan“ worden. Im weiteren Verlauf der Erzählung stellt sie diese Erfahrung dann als eine von mehreren dar:

„Also, (.) ich konnte nicht verstehen, [...] Auf jeden Fall, die Sachen, die ich da erlebt habe, die haben mich so beschäftigt, dass die mich auch zu Hause beschäftigt haben und dass ich nachts nicht mehr schlafen konnte. "11

\footnotetext{
9 Interview Linda, Zeilen 409-422.

10 Interview Linda, Zeilen 422-431.

11 Interview Linda, Zeilen 432-438.
} 
Linda erzählt, dass die aus ihrer Sicht indifferente Haltung gegenüber der alten Frau - ihre Sorge im Gegensatz zur vermeintlichen Teilnahmslosigkeit der hauptamtlichen Kraft - sie länger und intensiv beschäftigt habe. Sie habe nicht verstehen können, dass gegenüber den alten Menschen nicht mehr Empathie herrsche und habe deswegen den Sinn ihres Engagements in diesem Kontext in Frage gestellt. Dabei wird die Empörung auch erzählt als eine über das Engagement selbst hinausgreifende Auseinandersetzung mit der Organisation und der Pflegearbeit. Linda fasst dann zusammen:

„Für mich war dann alles so ungerecht wieder, [...] weil ich so sah, wie die Menschen/Ich meine, das Heim kann da selber auch nicht für. Weil, die kriegen ja/die kriegen ja gesagt, wie viel' Leute sie einstellen dürfen. Aber man sieht, dass es da einfach an Kräfte fehlt. Man kann so Demenzkranke ganz schön beschäftigen. Also, die kann man ja, wirklich Sinnvolles mach/also mehr Lebensqualität geben. Nur, das Personal muss dafür vorhanden sein. Oder noch nicht mal Personal, Menschen, die einfach auch ein Gespür dafür haben und sich einfach mal ein paar Stunden am Tag mit diesen Menschen beschäftigen. Entweder mal draußen an der frische Luft fahren oder auf kreativem Gebiet oder so was. Und die können dann auch gesund müde werden. Also, da braucht man keine Medikamente." 12

Linda rahmt das, was sie beobachtet, schließlich als ein Beispiel für eine allgemeine Ungerechtigkeit (,alles“). Mit ihrem Gegensatz von „gesund müde machen“ einerseits und Medikation andererseits verweist sie fast schon idealtypisch auf den Gegensatz zwischen einem menschlichen Umgang und einer technischen Versorgung in der Pflege - einem Gegensatz, den sie, so unsere Lesart, eben nicht auf diese eine Einrichtung beschränkt. Ihren Widerspruch adressiert sie dabei sowohl an die Ebene der Einrichtung als auch an eine nicht mehr von ihr beeinflussbare Ebene, auf der entschieden werde, welche finanziellen Mittel Altersheimen zur Verfügung stehen. Sie kritisiert das Personal vor Ort, dem „ein Gespür“ für die Menschen fehle, ohne dabei eine bestimmte Person im Auge zu haben. Sie kritisiert aber auch in einem breiteren Zusammenhang: demjenigen des politisch organisierten Pflegesystems mit machtvollen Interessengruppen. Die Rekonstruktion der Entwicklung der Erzählung zeigt somit, wie Linda mit einem konkreten Beispiel beginnt und bei der Schilderung einer allgemeinen Ungerechtigkeit endet.

Im Vergleich mit anderen Interviews erweisen sich sowohl der Aufbau der Passage als auch der erzählte Gehalt als typisch: Der Widerspruch taucht in der Erzählung nicht von Anfang an auf, sondern erst mit konkreten Erfahrungen. Diese werden exemplarisch für die erlebten Missstände erzählt und erhalten aufgrund der detaillierten Schilderungen, der engagierten Erzählweise sowie ihrer Stellung im Interview eine besondere Bedeutung. Mit den konkreten Episoden sehen sich die Engagierten in ihren Werthaltungen herausgefordert. Sie setzen sich mit diesen Schilderungen gleichzeitig in Gegensatz zu dem, was sie im Engagementfeld erleben. Weiterhin versucht Linda, dem Interviewer zu verdeutlichen, wie weit ihre Position als Engagierte und diejenige der Altenstiftorganisation auseinanderliegen. Ihr Widerspruch

12 Interview Linda, Zeilen 458-474. 
richtet sich im Resümee der Erzählung nicht in erster Linie an konkrete Personen oder Einrichtungen, sondern an allgemeinere gesellschaftliche Zustände.

\subsection{Typische Aspekte des Widerspruchs}

Nach dieser Betrachtung der Entstehung des Widerspruchs im Engagementverlauf wird nachfolgend die Perspektive gewechselt: Widerspruch als spezifisches Engagiertenwissen und Engagementerfahrung ist ein Begriff für ein aus dem empirischen Material entwickeltes Phänomen, das durch vier spezifische Aspekte gekennzeichnet ist. Diese werden nachfolgend weniger materialintensiv dargestellt.

\subsubsection{Starke Emotionen}

Lindas Erzählungen über ihren Widerspruch an dem, was sie im Altersheim erlebte, sind durch starke Emotionen, insbesondere durch Empörung, aber auch durch Mitleid mit den Betreuten und einem Leiden an dem, was sie sehen musste, geprägt. Im Vergleich der Schilderungen über Widerspruchserfahrungen finden wir Emotionen in unterschiedlichen Ausprägungen vor. Einige Interviewte erzählen überhaupt sehr emotional: Sie zeigen sich freudig während des Interviews und lachen oder äußern sich eher zornig oder wütend und formulieren mit einer gewissen Schärfe. ${ }^{13}$ Andere hingegen nutzen nur passagenweise eine dezidiert kräftige Ausdrucksweise, welche uns zu der Interpretation führt, dass die so geschilderte Engagementsituation Emotionen hervorrief. Ansonsten wirkt die letztgenannte Gruppe während der Interviews aber eher ruhig und emotionslos. Sowohl die Erzählweisen als auch die erzählten Erfahrungen sind also beide in unterschiedlichem Maß mit Emotion verwoben.

Die Erzählung von Linda ist eine, die fast durchgängig durch eine emotionale Erzählweise wie auch durch Schilderungen über Emotionen geprägt ist. Ein kurzer Vergleich mit zwei anderen Interviews, welche zur Gruppe der eher emotionslos Erzählenden gehören, macht deutlich, wie hier der Widerspruch emotional gefärbt ist: Ein Engagierter ist zurückhaltender hinsichtlich seiner Darstellung von Emotionen gegenüber den hauptamtlichen Kräften. Er spricht eher kühl über das Unbehagen mit der Verlagerung von Sorgetätigkeiten aus den Familien in vergleichsweise anonyme Organisationen, welche durch Ökonomisierung und Effektivierung geprägt seien. Aber wenn er davon spricht, wie er seine Erfahrungen im Altersheim mit anderen geteilt habe, dann umschreibt er die Situation mit den Worten: ,mir tut weh diese Situatione“ 14 oder ,ich habe tiefe Schmerz in meine Herz"15. Ein anderer Engagierter, dessen Erzählweise sehr stark rational und distanziert wirkt, findet beim Reden über behördliche Fehltritte im Umgang mit den von ihm betreuten Asylbewerber*innen starke Worte, die aufgrund ihres adressierenden Charakters den Eindruck erwecken,

\footnotetext{
13 In diesen Fällen mag einem eine frühe und zugegebenermaßen umstrittene Idee von Fritz Schütze (1984) einfallen, wonach Erzählkonstitution und Erfahrungskonstitution homolog seien: Gerade in den Stegreiferzählungen narrativer Interviews hätten Sozialforscher*innen, so Schütze, einen Text zur Verfügung, der dem faktischen Handeln und Erleiden am nächsten stehe (vgl. Schütze 1984; sowie zur Kritik: Bude 1985).

14 Interview Francesco, Zeilen 889-890.

15 Interview Francesco, Zeilen 205-206.
} 
im Moment der Datenerhebung zu gelten: „Wissen Sie“, sagt er, „da packt einen die Wut". Diese unterschiedlichen Arten des Erzählens von Emotionen ließen sich als Dokument eines Wissens deuten, welches nicht einfach rational da ist, sondern eben auch tieferliegende Überzeugungen tangiert.

Unsere Annahme lautet an dieser Stelle, dass die Engagierten Kritikwürdiges nicht nur einfach gesehen haben und während des Interviews rational benennen, sondern dass es für die Analyse notwendig ist, der Emotionalität in den einzelnen Erzählungen nachzugehen. Ausgehend von der idealtypischen Unterscheidung Max Webers (2014) zu den „Arten des sozialen Handelns“ (Weber 2014, S. 15f.) ist affektuelles Handeln lange Zeit in den Sozialwissenschaften als irrational gegenüber etwa einem zweckrationalen Handeln vernachlässigt worden. Wir wollen demgegenüber darauf hinweisen, dass die Emotionalität in den geschilderten Passagen als Hinweis auf eine Art Krise oder turning point gedeutet werden kann: Scheinbar wurden die Engagierten an solchen Stellen mit der Erfahrung konfrontiert, eine von ihnen für gut befundene Handlungsroutine so nicht mehr vollziehen zu können. Mit dem, was sie gesehen haben, so lesen wir ihre Schilderungen, können sie nicht mehr einfach weitermachen wie zuvor. An dieser Stelle wird deutlich, dass hier nicht lediglich die eigene Motivation zum Engagement in Frage steht und auch nicht eine Unsicherheit hinsichtlich der eigenen Rolle oder Aufgabe besteht, wie es die Engagementforschung bisweilen interpretiert, sondern dass hier auch fundamentale Überzeugungen im Engagement tangiert sind.

Dass der Widerspruch auf diese Weise emotional erzählt wird, hängt in unserer Interpretation auch davon ab, dass die Engagierten zuvor von starken positiven Erfahrungen erzählt haben, die sie an ihre Klient*innen oder bestimmte Selbstverständnisse ihrer Tätigkeiten gebunden hätten. Unsere These ist, dass gerade dann, wenn Engagierte eine besondere Nähe zu Menschen aufgebaut haben, sie deren Schicksal auch besonders berührt. Dies gilt insbesondere dann, wenn sie den Eindruck haben, dass mit diesen Menschen ungerecht oder unmenschlich umgegangen wird.

\subsubsection{Einsamer Widerspruch}

So emotional die Befragten ihren Widerspruch dem Interviewer gegenüber auch artikulieren, so wenig erzählen sie von konkreten Aktionen, in denen sie Verbesserungsvorschläge vorbrachten, Kritik äußerten oder auf andere Art und Weise ihren Widerspruch in den Einrichtungen explizierten. Nur ganz selten ist in den Interviews tatsächlich auch mal die Rede von erfolgreichen Veränderungen, etwa dem Einsetzen eines progressiven Vereinsvorstands. Dies führen wir zunächst darauf zurück, wie dieser Widerspruch konkret sozial situiert wird: Wir haben beobachten können, dass die Engagierten ihr Widersprechen als einen einsamen Akt darstellen. Auch im Interview mit Linda wird dies deutlich: Linda erzählt sich sowohl in ihrer Empörung als auch in ihrer Beschwerde gegenüber der zuständigen Hauptamtlichen und schließlich im Aushalten von Unmut zuhause nach der Tätigkeit als einzelne Person. Sie berichtet an keiner Stelle von anderen Engagierten (oder auch Hauptamtlichen), die sie in welcher Weise auch immer unterstützt hätten. Dies erweist sich im Vergleich der Erzählungen als typisch. 
Über den Grund für diese Darstellungsweise können wir auf Grundlage unseres Materials keine eindeutige Aussage treffen: Manche Engagierte erzählen etwa davon, dass sie Mitengagierte nicht als in der Lage gesehen haben, Widerspruch zu äußern oder Verbesserungen umzusetzen, sei es, weil die anderen Engagierten zu alt, zu unkritisch oder zu sehr mit dem Engagement verbunden gewesen wären. Andere haben über den gesamten Interviewverlauf von sich das Bild einer Einzelkämpfer*in gezeichnet, die ihr Engagement eher selbstgenügsam verrichtet. Auch wenn dies nicht den gängigen Vorstellungen von Engagement entspricht, in dem den Engagementsurveys zufolge doch Geselligkeit und Spaß gesucht werde (Simonson et al. 2017, S. 426 ff.), scheint uns diese Erzählung typisch zu sein, auch in Fällen unseres Samples, die weniger auf Widerspruch hinauslaufen: Selten wird in den Interviews von anderen Engagierten gesprochen, mit denen gemeinsam Engagementprozesse erörtert wurden. Verbesserungsvorschläge werden nicht erzählt als etwas, was im Feld breit artikuliert wird, im Gegenteil: Die Engagierten scheinen nicht nur in ihrem Engagement eher allein mit den Klient*innen zu sein - sondern sie bleiben auch in ihrem Widerspruch eher isoliert. Insofern wird von Widerspruch nicht erzählt als Ausgangspunkt einer Koalitionsbildung z. B. mit anderen Engagierten oder auch mit Hauptamtlichen. Das Fehlen weiterer Interaktionspartner*innen mag erklären, warum die von uns befragten ehemaligen Engagierten nicht im Engagement verbleiben und dort weiteren Widerspruch initiieren.

\subsubsection{Diffuse Adressatenschaft}

Von der sozialen Situierung eines Widerspruchs und der Frage, ob er überhaupt stattfindet, unterscheidet sich zusätzlich die Adressatenschaft, die die Interviewten in ihren Erzählungen für ihren Widerspruch durchspielen. Häufig ist ihnen nicht klar, an wen sie sich wenden sollen, weil die Personengruppe möglicher Ansprechpartner*innen ihnen entweder als zu diffus oder als nicht verantwortlich erscheint. Auch dies wird in der Analyse der Erzählung von Linda deutlich. Sie sagt im Interview, dass das Heim selbst die Betreuungsangebote nicht festlegen könne, da es von der Finanzierung durch übergeordnete Institutionen abhängig sei („Ich meine, das Heim kann da selber auch nicht für. Weil, die kriegen ja/die kriegen ja gesagt, wie viel' Leute sie einstellen dürfen."). Der Gegenhorizont einer solchen Darstellung wäre ein Heim, welches selber tatsächlich auch darüber entscheiden könnte, wie es sich personell aufstellt. In einem solchen Heim wäre die Einrichtungsleitung dann auch tatsächlich ansprechbar und wirksam zu kritisieren. Die von Linda vorgenommene Einschränkung verweist demgegenüber darauf, dass Linda in ihrer Kritik durchaus auch über Verantwortlichkeit nachdenkt - ihrem direkten Gesprächspartner diese aber abspricht.

Ganz ähnlich wird dieses Prinzip der Verantwortlichkeitsüberlegung in der Erzählung der Engagierten Semra deutlich. Sie wollte sich im Besuchsdienst eines kirchengeführten Krankenhauses engagieren. Dieses Engagement sei gescheitert, so erzählt Semra, weil eine Mitengagierte ein Kopftuch trug, was den Kleidungsvorschriften des Krankenhauses widerspreche. Der Widerspruch, der das zentrale Thema der Erzählung bildet, richtet sich gegen eine solche Ausgrenzung aufgrund eines Kopftuches. Bemerkenswert ist dabei, dass von Semra drei verschiedene Ebenen 
bzw. Zielgruppen der Ansprache für den Widerspruch genannt werden: Zunächst berichtet sie ausführlich von einem Gespräch mit der konkreten Dame, die ihr die Nachricht überbringt. Sie schildert lebendig, wie sie die Ablehnung des Kopftuchs in diesem Gespräch hinterfragt: „Äh Moment [...]. Äh, warum nicht? Das ist eine ehrenamtliche Sache. [...] Warum, weshalb?"16. Indem sie dann jedoch ein Gespräch mit „dem Obersten“17 des Krankenhauses fordert, betont sie die Leitungsebene der Einrichtung als verantwortlich für die Kleidungsvorschrift und somit als Adressatin des Widerspruchs. Schließlich beschreibt sie Grenzziehungen gegenüber Muslim*innen ausführlich als ein gesamtgesellschaftliches Problem, das hier im konkreten Engagementfeld seinen Ausdruck finde: ,dass ein Kopftuch so viel ausmacht, ist traurig“18, sagt sie. „Das ist wieder diese Diskriminierung.“"19 Dass Semra kein Gespräch mit „dem Obersten“ bekommen hat, sei ihr mittlerweile egal: „Ist mir jetzt auch ganz egal. Will ich auch nicht mehr." ${ }^{20}$ Dass sie ihre dem Interviewer gegenüber so heftig artikulierte Kritik der Einrichtung gegenüber nicht weiterverfolgt, erklären wir uns mit ihrer gesellschaftlichen Rahmung der konkreten Situation: Der konkreten Dame, die letztlich keine Entscheidungsgewalt hat, kann sie widersprechen. An wen hätte sie sich aber wenden sollen, um einer gesellschaftlichen Diskriminierung zu widersprechen?

Diese Erzählweise zeigt sich auch in der Schilderung einer weiteren Engagierten über eine fehlende Adresse für den Widerspruch am Verschwinden gespendeter Kleidung aus einer Kleiderkammer. Von der Engagierten werden zunächst die konkrete Kleiderkammer und dann der lokale Wohlfahrtsverband als verantwortlich ausgemacht. Schließlich rahmt die Engagierte ihr Unbehagen mit einer Fernsehdokumentation, in der die Unterschlagung von gespendeten Kleidern als ein Fehlverhalten ganzer Wohlfahrtsorganisationen diskutiert worden sei. Sie widerspricht somit gegen eine Praxis, die sie letztendlich nicht nur als Praxis vor Ort, sondern auch als allgemeine Praxis von unterschiedlichen Wohlfahrtsorganisationen darstellt.

Im Vergleich zwischen den Interviews finden sich typische Merkmale in den möglichen Adressierungen von Widerspruch: In den Erzählungen tauchen zwar immer wieder auch konkrete Personen auf, denen aber letztendlich nicht die Verantwortung für die verbesserungswürdigen Aspekte im Engagement zugeschrieben wird. Das heißt, es werden oftmals gesichtslose, abstrakte und unpersönliche Strukturen, Organisationen, Abläufe etc. kritisiert. Konkrete Akteur*innen werden dabei in den Darstellungen als Rädchen im System dargestellt. Entsprechend werden sie in den Interviews meist nur kurz und unpersönlich beschrieben: die Ordensschwester im Altenstift, der Sachbearbeiter im Landesbüro der Wohlfahrtsorganisation usw. Indem ihnen nicht zugetraut wird, für eine Praxis auch tatsächlich ansprechbar und verantwortlich zu sein, fallen sie aus der Wahrnehmung möglicher Adressat*innen von Widerspruch heraus. Einige Engagierte betonen ganz explizit, dass es ihnen bei ihrem Widerspruch nicht um eine spezifische Einrichtung gehe, sondern um einen

\footnotetext{
16 Interview Semra, Zeilen 53-57.

17 Interview Semra, Zeile 61.

18 Interview Semra, Zeilen 115-116.

19 Interview Semra, Zeilen 128-129.

20 Interview Semra, Zeilen 63-64.
} 
Zustand, den es an vielen Orten gebe. Ein Engagierter stellt z.B. fest, dass sich prekäre Pflegeverhältnisse in allen Einrichtungen fänden, ,egal von wem, Caritas, Diakonie, AWO“. Bei anderen Engagierten bleibt diese Verallgemeinerung von Widerspruch eher implizit, wie etwa in dem genannten Kopftuchstreit. In Ergänzung des einleitend geschilderten Forschungsstandes lässt sich somit sagen, dass es für den Verbleib im bürgerschaftlichen Engagement nicht nur darauf ankommt, ob es Kommunikation zwischen Haupt- und Ehrenamtlichen gibt. Vielmehr kommt es darauf an, ob die Engagierten den Hauptamtlichen zuschreiben, tatsächlich einen Unterschied machen zu können.

\subsubsection{Nicht-artikulierter Widerspruch}

Einige Engagierte äußern ihren Widerspruch gar nicht explizit gegenüber maßgeblichen Verantwortlichen, etwa einer für Ehrenamtliche zuständigen sozialpädagogischen Fachkraft. Sie denken zwar darüber nach, mit der Einrichtungsleitung zu sprechen, tun es dann aber nicht. Nur wenige berichten davon, dass sie Gespräche hatten, die dann jedoch ohne Erfolg blieben. So erzählt eine Engagierte, dass ihr von einem solchen Gespräch abgeraten worden sei, und zwar von anderen Mitarbeiterinnen, weil es keinen Sinn mache: „Mach Dir keine Mühe“. Weiterhin haben während der Vorbereitungen unserer Feldkontakte und nachträglich bei der Vorstellung der Ergebnisse zahlreiche Hauptamtliche der Wohlfahrtsverbände beklagt, dass sie von der Unzufriedenheit der Engagierten selten etwas mitbekommen, eben weil sie kaum angesprochen werden. Abwanderung sei dem Widerspruch vorgezogen worden und der kritische Impuls in die Organisation sei unterblieben.

Als Erklärung für das Auslassen eines expliziten Widerspruchs lässt sich auf der Grundlage unserer Ergebnisse neben dem Fehlen von Koalitionen im Engagement (Abschn. 4.2) und konkreten Ansprechpartner*innen (Abschn. 4.3) noch ein dritter Punkt anfügen: Wenn Engagierte etwa in der Pflege ihre Ideale vorstellen, dann beziehen sie sich auf Normen wie die Verantwortung gegenüber älteren Generationen und Ideale wie Wärme und Geborgenheit. Das scheinen uns Bilder zu sein, die eher einen familiären Rahmen des Älterwerdens implizieren. Von den Wohlfahrtsverbänden wird hingegen das Bild gezeichnet, dass diese eine abstrakte Dienstleistungsbeziehung zu deren Klient*innen hätten, in der es um eine Art Grundversorgung gehe (an Essen, an Medikamenten und an Körperpflege). Das Handeln der Engagierten wird hier unter Verweis auf einen anderen Kontext gerechtfertigt (nämlich Familie), als dies für das Handeln des Verbandes getan wird (nämlich Dienstleistungsbeziehung/Markt). Somit lässt sich beobachten, dass die Engagierten für ihr Handeln eine andere Art Rechtfertigung bzw. einen anderen Rechtfertigungskontext in Anspruch nehmen, als sie den Verbänden zuschreiben. ${ }^{21}$ Insofern scheinen die Engagierten nicht davon ausgegangen zu sein, dass sich ein Widersprechen lohne, weil aus ihrer Perspektive keine gemeinsame Grundlage der Rechtfertigung einer bestimmten Engagementpraxis bestanden habe. In diesem Zusammenhang ist auch bedeutsam, dass einige Engagierte erzählen, dass sie sich mit Partner*innen, Freund*innen oder an-

21 Mit Luc Boltanksi und Laurent Thévenot (2007) ließe sich in diesem Zusammenhang von unterschiedlichen Rechtfertigungsordnungen sprechen. 
deren nahestehenden Personen über das Engagement besprochen hätten. Es erschien ihnen möglicherweise plausibler, den Widerspruch mit einer privat nahestehenden Person zu teilen, mit der man gewisse Werte teilt, als mit einer Organisation, der man dies nicht unterstellt.

Zusammenfassend stellen wir für das Phänomen Widerspruch fünf konstitutive Bestandteile fest, die wir aus der jeweiligen Fallbetrachtung und der fallvergleichenden Analyse entwickeln konnten: Widerspruch als Haltung entwickelt sich ausgehend von starken Erfahrungen, die von den Engagierten verallgemeinert werden. Er wird emotional erzählt, was darauf verweist, dass die kritisierten Zustände grundlegende Überzeugungen der Engagierten betreffen. Er wird im Kreis der Engagierten scheinbar nicht derart breit artikuliert, als dass sich Koalitionen bilden könnten; vielmehr bleiben Widersprechende eher allein. Weil der Widerspruch sich zwar an konkreten Erfahrungen entzündet, aber allgemeine Zustände anspricht, fehlt eine konkrete Adressatenschaft. Die Engagierten sehen keine gemeinsame moralische Grundlage zwischen ihnen und dem Engagementfeld und der Widerspruch wird dort nicht artikuliert.

\section{Was über das Engagement hinausgeht. Widerspruch als Positionierung}

Entscheidend für die im Folgenden vorgestellte theoretische Rahmung unserer Ergebnisse ist somit folgende Beobachtung: Die befragten Engagierten erzählen Geschichten über starke Erfahrungen während des Engagements, aber auch Geschichten, in denen politischen und/oder moralischen Werten eine besondere Rolle zugewiesen wird. Das heißt, die ehemaligen Engagierten erzählen oft und ausführlich über Solidarität, über Gerechtigkeit, über einen würdevollen Umgang mit alten Menschen, über Transparenz oder Wertschätzung. Indem sie eine Geschichte über ihr ganz spezifisches Engagement erzählen, etwa über ihre konkreten Aufgaben und über ihre Interaktionen und Beziehungen im Engagement, erzählen sie auch Bindungserfahrungen an und Geltungsbekenntnisse über Werte. Dies verstehen wir nicht lediglich als einseitige Anpassungen an Rollen oder Organisationen, sondern auch als Prozesse der Selbstbildung seitens der Engagierten.

Diese Beobachtung anhand unseres Datenmaterials korrespondiert mit Überlegungen, die der Sozialphilosoph Hans Joas (1997) über die Geltung und Thematisierung von Werten vorgestellt hat. Als Handlungstheoretiker und Pragmatist konzipiert Joas (1997) Werte weniger als teleologische Leitmotive von Handlungssystemen, sondern als affektiv intensive und subjektiv evidente Vorstellungen des Guten für die jeweils handelnde Person. Solche Geltungsüberzeugungen des Guten - mit Joas ließe sich von Wertbindungen sprechen - entstammen neben der Sozialisation ganz zentral dem Erleben und Interpretieren von außergewöhnlichen Erfahrungen (vgl. Joas 1997, S. 10). Eine Wertbindung äußere sich maßgeblich in starken moralischen Gefühlen, etwa, dass diese Handlung gut und richtig, jene aber falsch sei. Sie sei dadurch konstitutiv für die personale Identität einer Person. Insofern Werte Ausdruck einer bestimmten praktischen Erfahrung und deren Interpretation sind, sei die Art 
und Weise des Redens über Werte und Wertbindungen eben nicht die allgemeine rationale Rechtfertigung der Fundamentalphilosophie, sondern die Erzählung. ${ }^{22}$

Folgt man einer solchen Argumentation über das Entstehen von Wertbindungen aus konkreten Erfahrungen heraus, dann besitzt man auch eine Erklärung über die Wandelbarkeit von Identitäten. Diese scheint uns notwendig zu sein, um die Neuorientierung von einer engagementinteressierten Person zu einer dem Engagementfeld widersprechenden Person verstehen zu können. Der Prozess der Interpretation maßgeblicher Erfahrung stellt demnach für die Sinnhaftigkeit neuer Handlungsroutinen und ihrer moralischen Orientierungen den maßgeblichen biografischen Bezugspunkt dar. Auf diese Weise werden Erfahrungen zu Bestandteilen einer narrativen Identität, ${ }^{23}$ das heißt einer erzählerischen Sinnhaftmachung von Welt, mit der sich die Engagierten in Beziehung zu ihrem Engagementfeld und den maßgeblichen Akteur*innen setzen. Wir denken Widerspruch in diesem Sinne als Teil eines moralischen Wissens, das sich anhand von starken Erfahrungen im bürgerschaftlichen Engagement entwickelt. In diesem Sinne geht Widerspruch mit sich wandelnden Selbstverpflichtungen dem Engagementfeld gegenüber einher.

Die letztgenannte Einsicht hat seine Gültigkeit nicht nur für die jeweils engagierte Person, also nicht nur auf der Mikroebene, sondern sie lässt sich mithilfe der Idee der Positionierung auch gut abstrahieren: Die Artikulation von Verbesserungsvorschlägen, Kritik oder Unzufriedenheit ist auch eine Positionierung, die noch länger nach dem Engagementende wirkt, sowohl für Engagierte wie auch für Organisationen oder Engagementtätigkeiten. Das heißt, dass die Engagierten sich auch nach Beendigung des Engagements als politische bzw. moralische Engagierte darstellen, und zwar indem sie ihrer Engagementstelle oder Wohlfahrtsorganisation Eigenschaften zuschreiben, die sie als gegensätzlich zu ihrer eigenen Werthaltung beschreiben.

Unter Positionierung verstehen wir mit Gabriele Lucius-Hoene und Arnulf Deppermann (2004) ,diskursive Praktiken, mit denen Menschen sich selbst und andere in sprachlichen Interaktionen auf einander bezogen als Personen her- und darstellen, welche Attribute, Rollen, Eigenschaften und Motive sie mit ihren Handlungen in Anspruch nehmen und zuschreiben“ (Lucius-Hoene und Deppermann 2004, S. 168; vgl. auch Varga und Munsch 2014). Der Begriff der Positionierung ist passend zu der oben entwickelten Perspektive der Wertbindung, weil es sich bei Positionierungen ebenfalls um narrative Identitäten handelt, also um Erzählungen über ein Eigenes im Kontext unterschiedlicher zeitlich-räumlicher Erfahrungen und Einflüsse. Auch Joas betont für den Prozess der Wertbindung das Abwägen unterschiedlicher

\footnotetext{
${ }^{22}$ Bei den Arbeiten von Hans Joas gilt es zu beachten, dass diese nicht primär für eine Fundierung von empirischen Studien formuliert wurden. Vielmehr stellen sie zunächst als sozialphilosophische bzw. sozialtheoretische Argumentationen eine Alternative zu anderen erkenntnistheoretischen und moralphilosophischen Ansätzen der nachmetaphysischen Begründung von Werten dar (vgl. Joas 2002, 1997, S. 10-36). Gleichwohl braucht empirische Forschung eine sozialtheoretische Grundlegung, weswegen wir Joas “ Argumentation auch für die empirische Analyse nutzen.

23 Der Begriff der narrativen Identität meint das Herstellen eines kohärenten Selbst mittels Erzählung, auch wenn die erzählende Person hierzu höchst unterschiedliche konstitutive Erfahrungen verknüpft. Zum Begriff der narrativen Identität vgl. Paul Ricœur (1996), auf den Joas sich bezieht, sowie Jürgen Straub (2004), der sich ebenfalls auf Ricœur und Joas bezieht.
} 
Deutungsangebote. Selbstpositionierung geht somit immer auch mit einer Fremdpositionierung einher. Indem die Engagierten sich in Bezug auf bestimmte Werte oder Aufgaben selbst positionieren und die Wohlfahrtsverbände auf konträre, mit ihren eigenen Werten nicht kompatible festlegen, verweisen sie auf ein implizites Wissen über bestehende Spannungsfelder. Unter Rückgriff auf die Dokumentarische Methode ließe sich gar die These aufstellen, dieses Denken in Spannungsfeldern sei selbst ein Produkt (ein Dokument) des Handlungsfeldes Wohlfahrt, welches Hilfe im Spannungsfeld zwischen Effizienz und Humanität organisieren muss ${ }^{24}$.

In der Erzählung von Linda wird diese Selbst- und Fremdpositionierung sehr deutlich: Linda beschreibt sich an vielen Stellen des Interviews sehr eindringlich als eine Sorgende, welche es schafft, in eine „schöne“ Beziehung zu den alten Menschen $\mathrm{zu}$ treten. Ihr gelinge es mit viel Aufmerksamkeit, viele kleine Dinge im Alltag dieser Menschen zu verschönern. Wesentlich für diese Selbstdarstellung sind dabei konkrete Schilderungen, z. B. über das gemeinsame Filzen mit einer alten Dame. Ihre Beschreibung der Praxis der Hauptamtlichen im Altenheim, welche die alten Menschen mit Medikamenten ruhigstellen würden, positioniert diese in deutlichen Gegensatz zum eigenen Engagement mit den alten Menschen an der frischen Luft oder beim gemeinsamen Basteln.

In ähnlicher Weise kritisiert ein Engagierter, dass die alten Menschen im Altersheim nur als „Kassenpatient[en]“25 gesehen und allein gelassen würden. Er verdeutlicht den Kostendruck mit dem eindringlich erzählten Beispiel, wie die alten Menschen beim Abendbrot z. B. nach einer zweiten Scheibe Brot extra fragen müssten - und das, nachdem sie so lange gearbeitet hätten. „Das geht nicht“, sagt er, ,ist gegen meine Moral“26. Seine Moral, erzählt er, ist eine, nach der in den Familien gemeinsam nach den alten Menschen geschaut werde. Er positioniert den Wohlfahrtsverband im Gegensatz dazu als unmoralischen Akteur, der sich einer kulturellen Tradition der Organisation von Pflege entziehe und stattdessen diesen Bereich ökonomisiere. Die Selbstpositionierung in Gegensatz zu den Wohlfahrtsverbänden operiert in diesem Sinne mit der Darstellung einer Handlungslogik, die sich an der Kostenvermeidung in der Pflege orientiere. Indem der Engagierte dieses relationale Gefüge aufzeigt, lässt er auch einen Einblick in seine zukünftigen Ambitionen als Engagierter zu. Es scheint unwahrscheinlich, dass er sich hier jemals wieder freiwillig und unentgeltlich einbringt. Seiner Erzählung zufolge gründet er nach seinem Engagementende einen Arbeitskreis, in dem er die Indienstnahme von bürgerschaftlichem Engagement thematisiert und insbesondere Migrant*innen davor warnt, dieses als Möglichkeit eines Zugangs zur Erwerbstätigkeit zu betrachten.

Die erzählten Positionierungen der anderen und die Selbstpositionierung durch das Bekenntnis zu bestimmten Idealen und Werten scheinen uns derart wirkmächtig zu sein, dass sie auch über das Engagement hinaus Bestand haben. Dabei bedeutet die Selbstpositionierung in Gegensatz zum Engagementfeld nicht, dass sich die Engagierten nachfolgend nicht mehr engagieren würden. Sie schließen nur ein En-

${ }^{24}$ Diese Perspektive ist allerdings eine durchaus bekannte und breit diskutierte (vgl. dazu Haubner 2017; Browne 2010).

25 Interview Francesco, Zeile 168.

${ }^{26}$ Interview Francesco, Zeile 197. 
gagement aus, das unter diesen kritisierten Bedingungen stattfinden würde. Zusammenfassend wird somit deutlich, wie stark die Selbst- und Fremdbeschreibung über den Bezug auf Normen funktioniert. Sie produziert Rahmungen, innerhalb derer das eigene und das Handeln anderer als (nicht) sinnvoll erscheinen ${ }^{27}$. Insofern somit im Engagement Schemata und Erwartungshaltungen (re-)produziert und später im Interview reflektiert werden, sind Interaktionen von Engagierten und ihren Verbänden nicht ausschließlich nur als Prozesse der Mikroebene zu verstehen.

\section{Fazit}

Die Rekonstruktion der Erzählungen zeigt zunächst allgemein, dass Engagierte nicht einfach Aufgaben übernehmen, ohne nachzufragen oder sich selbst dazu Gedanken zu machen. Engagierte setzen sich vielmehr kritisch mit den Bedingungen auseinander, die sie vorfinden und nach und nach beobachten. Sie entwickeln eine eigene (durchaus politische) Position in Abgrenzung zum Engagementfeld, die auch noch nach ihrem Austritt wirksam bleibt und in Erzählungen artikuliert wird. Insofern kann bürgerschaftliches Engagement in Wohlfahrtsverbänden als ein Ort der Politisierung und der informellen politischen Bildung beschrieben werden.

Über diese etwas allgemeine Feststellung hinaus zeigen die Interviewanalysen, welche spezifischen Aspekte für das Widersprechen der (ehemaligen) Engagierten typisch sind. Zum einen entwickeln Engagierte im Verlauf ihres Engagements eine Engagementkarriere mit konkreter werdenden Vorstellungen über ihr Engagement, aber auch einer Intensivierung und (Re-)Aktivierung eigener moralischer Standards. Zum anderen erkennen die Engagierten, dass das, wogegen sie widersprechen, nicht auf eine einzelne Einrichtung, schon gar nicht auf eine einzelne Fach- oder Leitungskraft beschränkt ist. Personen und Ereignisse werden in den Erzählungen eher als einzelne Beispiele für übergreifende Zustände dargestellt. In diesem Zusammenhang wird deutlich, dass den Engagierten die Adresse des Widerspruchs unklar bleibt. Die Engagierten verorten die Verletzung ihrer grundlegenden Werte zum Teil auf einer derart abstrakt bleibenden systemischen Ebene, dass ihnen dies kaum Potenziale zur konkreten Situationsverbesserung ermöglicht habe. Außerdem fehlt in ihren Erzählungen über den Widerspruch die Unterstützung anderer ebenso wie eine gemeinsam geteilte Rechtfertigungsordnung. Dieses Fehlen sowohl von konkreten Adressat*innen als auch von Mitstreiter*innen mag dazu geführt haben, dass Widerspruch nicht als eine Protestaktion erzählt werden kann. Widerspruch ist in unserem Material somit zunächst eine individuelle Positionierung, die zwar zum Teil mit großer emotionaler Kraft erzählt wird, deren Adressat*innenkreis sich jedoch auf den Interviewer sowie Freund*innen und Partner*innen beschränkt, also auf eine nichtöffentliche Sphäre.

Den von uns analysierten Widerspruch könnte man als eine affektiv starke narrative Positionierung der Engagierten gegen ein als eher diffus empfundenes Engagementumfeld beschreiben. Es ist ein Widerspruch, der v.a. das widersprechende

27 Der Sozialpsychologe Rom Harré und Kolleg*innen (2009), der die Positionierungsanalyse maßgeblich mitentwickelt hat, spricht in diesem Zusammenhang von Story-Lines. 
Subjekt verändert, weniger jedoch Organisationsroutinen, die es adressiert. Unsere Rekonstruktionen zeigen, dass Handlungslogiken der Wohlfahrtsorganisationen den Engagierten wenig empfänglich für Widerspruch zu sein scheinen. Diese These mag provokant erscheinen und durchaus auch mit vorherigen Ergebnissen in Konflikt stehen (etwa Hämel 2012, S. 101). Sie spiegelt aber das grundsätzliche Dilemma zivilgesellschaftlicher Organisationen wider, welche einerseits Effizienzerwartungen unterliegen, andererseits wesentliche Elemente des demokratischen Gemeinwesens sind (vgl. Dahme und Wohlfahrt 2007; Munsch 2005).

Was bedeutet das Gesagte nun für Wohlfahrtsverbände als Orte des Widerspruchs? Die Verbände haben in systemischer Perspektive als zivilgesellschaftliche Akteure in einer Sphäre neben Markt und Politik zunächst ein Mandat, Widerspruch zu äußern (etwa gegen eine unzureichend finanzierte Pflegepolitik). Sie bieten zugleich mit dem bürgerschaftlichen Engagement Räume und Erfahrungen für die Bildung und Stabilisierung kritischer Subjekte. Wohlfahrtsverbände werden von den dort engagierten Freiwilligen als Marktakteure wahrgenommen, die mit anderen sozialen Dienstleister*innen konkurrieren. Dieses Wissen über hybride Organisationen, welches die Dritte-Sektor-Forschung bereits länger formuliert hat (vgl. Hämel 2012; Evers 2010), entwickelt sich somit auch bei den Engagierten. Wenn den Engagierten die Wohlfahrtsverbände als routinierte Marktakteure erscheinen, die indifferent gegenüber dem Widerspruch der Engagierten sind, weil sie diese lediglich als ökonomische Ressourcen wahrnehmen, dann hat dies Konsequenzen sowohl für das politische Gemeinwesen als auch für die betroffenen Organisationen. Denn dann würde die politisierende Erfahrung eine individuelle bleiben und die öffentliche Artikulation von Widerspruch gerade da ausbleiben, wo sie der Idee der Zivilgesellschaft nach eigentlich seinen Platz haben sollte: im Engagement.

Open Access Dieser Artikel wird unter der Creative Commons Namensnennung 4.0 International Lizenz (http://creativecommons.org/licenses/by/4.0/deed.de) veröffentlicht, welche die Nutzung, Vervielfältigung, Bearbeitung, Verbreitung und Wiedergabe in jeglichem Medium und Format erlaubt, sofern Sie den/die ursprünglichen Autor(en) und die Quelle ordnungsgemäß nennen, einen Link zur Creative Commons Lizenz beifügen und angeben, ob Änderungen vorgenommen wurden.

\section{Literatur}

Allen, J.A., \& Mueller, S.L. (2013). The revolving door. A closer look at major factors in volunteers' intention to quit. Journal of Community Psychology, 41(2), 139-155. https://doi.org/10.1002/jcop. 21519. [Letzter Zugriff am 19. Juni 2018].

Bohnsack, R. (2010). Rekonstruktive Sozialforschung. Einfuihrung in qualitative Methoden (8. Aufl.). Opladen: Barbara Budrich.

Boltanski, L. (2010). Soziologie und Sozialkritik. Frankfurter Adorno-Vorlesungen 2008. Berlin: Suhrkamp.

Boltanski, L., \& Thévenot, L. (2007). Über die Rechtfertigung. Eine Soziologie der kritischen Urteilskraft. Hamburg: Hamburger Ed.

Browne, P. L. (2010). The dialectics of health and social care. Toward a conceptual framework. Theory and Society, 39(5), 575-591. https://doi.org/10.1007/s11186-010-9120-6. [Letzter Zugriff am 19. Juni 2018].

Bude, H. (1985). Der Sozialforscher als Narrationsanimateur. Kritische Anmerkungen zu einer erzähltheoretischen Fundierung der interpretativen Sozialforschung. Kölner Zeitschrift für Soziologie und Sozialpsychologie, 37(2), 327-336. 
Dahme, H.-J., \& Wohlfahrt, N. (2007). Aporien staatlicher Aktivierungsstrategien. Engagementpolitik im Kontext von Wettbewerb, Sozialinvestition und instrumenteller Governance. Forschungsjournal Soziale Bewegungen, 20(2), 27-39.

Dunn, J., Chambers, S. K., \& Hyde, M. K. (2016). Systematic review of motives for episodic volunteering. Voluntas, 27(1), 425-464. https://doi.org/10.1007/s11266-015-9548-4. [Letzter Zugriff am 19. Juni 2018].

van Dyk, S., \& Misbach, E. (2016). Zur politischen Ökonomie des Helfens. Flüchtlingspolitik und Engagement im flexiblen Kapitalismus. Prokla, 46(2), 205-227.

van Dyk, S., Dowling, E., \& Haubner, T. (2016). Für ein rebellisches Engagement. Blätter für deutsche und internationale Politik, 2/2016, 37-40.

Ehrhardt, J. (2011). Ehrenamt. Formen, Dauer und kulturelle Grundlagen des Engagements. Frankfurt am Main: Campus.

Evers, A. (2010). Zivilgesellschaft, Engagement und soziale Dienste. In T. Olk, A. Klein \& B. Hartnuß (Hrsg.), Engagementpolitik. Die Entwicklung der Zivilgesellschaft als politische Aufgabe (S. 282-302). Wiesbaden: VS.

Garner, J. T., \& Garner, L. T. (2011). Volunteering an opinion. Organizational voice and volunteer retention in nonprofit organizations. Nonprofit and Voluntary Sector Quarterly, 40(5), 813-828. https://doi.org/ 10.1177/089976401036618. [Letzter Zugriff am 19. Juni 2018].

Glaser, B. G., \& Strauss, A. L. (2005). Grounded Theory. Strategien qualitativer Forschung (2. Aufl.). Bern: Huber.

Graf, L. (2016). Freiwillig im Ausnahmezustand. Die ambivalente Rolle ehrenamtlichen Engagements in der Transformation des Asylregimes. Widersprüche, 36(3), 87-96.

Hämel, K. (2012). Öffnung und Engagement. Altenpflegeheime zwischen staatlicher Regulierung, Wettbewerb und zivilgesellschaftlicher Einbettung. Wiesbaden: Springer VS.

Harré, R., Moghaddam, F.M., Cairnie, T.P., Rothbart, D., \& Sabat, S. R. (2009). Recent advances in positioning theory. Theory \& Psychology, 19(1), 5-31. https://doi.org/10.1177/0959354308101417. [Letzter Zugriff am 19. Juni 2018].

Haski-Leventhal, D., \& Bargal, D. (2008). The volunteer stages and transitions model. Organizational socialization of volunteers. Human Relations, 61(1), 67-102. https://doi.org/10.1177/001872670708 5946. [Letzter Zugriff am 19. Juni 2018].

Haubner, T. (2017). Die Ausbeutung der sorgenden Gemeinschaft. Laienpflege in Deutschland. Frankfurt am Main: Campus.

Hirschman, A. O. (1974). Abwanderung und Widerspruch. Reaktionen auf Leistungsabfall bei Unternehmungen, Organisationen und Staaten. Tübingen: Mohr Siebeck.

Hirschman, A. O. (1984). Engagement und Enttäuschung. Über das Schwanken der Bürger zwischen Privatwohl und Gemeinwohl. Frankfurt am Main: Suhrkamp.

Hustinx, L. (2010). I quit, therefore I Am? Volunteer turnover and the politics of self-actualization. Nonprofit and Voluntary Sector Quarterly, 39(2), 236-255. https://doi.org/10.1177/0899764008328183. [Letzter Zugriff am 19. Juni 2018].

Joas, H. (1997). Die Entstehung der Werte. Frankfurt am Main: Suhrkamp.

Joas, H. (2002). Werte versus Normen. Das Problem der moralischen Objektivität bei Putnam, Habermas und den klassischen Pragmatisten. In M.-L. Raters \& M. Willaschek (Hrsg.), Hilary Putnam und die Tradition des Pragmatismus (S. 263-279). Frankfurt am Main: Suhrkamp.

Kewes, A. (2017). Engagementbeendigung in der Wohlfahrtspflege. Vom Suchen, Finden und Verlieren einer sinnvollen Tätigkeit. In S. Lessenich (Hrsg.), Geschlossene Gesellschaften. Verhandlungen des 38. Kongresses der Deutschen Gesellschaft für Soziologie, Bamberg, 2016. http:// publikationen.soziologie.de/index.php/kongressband_2016/article/view/512. [Letzter Zugriff am 02. Oktober 2017].

Lucius-Hoene, G., \& Deppermann, A. (2004). Narrative Identität und Positionierung. Gesprächsforschung, $5,166-183$.

Munsch, C. (2005). Die Effektivitätsfalle. Gemeinwesenarbeit und bürgerschaftliches Engagement zwischen Ergebnisorientierung und Lebensbewältigung. Baltmannsweiler: Schneider Verlag Hohengehren.

Nohl, A.-M. (2017). Interview und Dokumentarische Methode (5. Aufl.). Wiesbaden: Springer VS.

Ricœur, P. (1996). Das Selbst als ein Anderer. München: W. Fink.

Ripamonti, C. A., Pasquarelli, L., Ravasi, S., \& Sala, F.C. (2017). Dropout of hospital volunteers in Italy. Voluntas, 28(1), 44-68. https://doi.org/10.1007/s11266-016-9769-1. [Letzter Zugriff am 19. Juni 2018]. 
Rochester, C., Paine, E. A., \& Howlett, S. (2010). Volunteering and society in the 21 st century. Houndmills, Basingstoke: Palgrave Macmillan.

Roth, R. (2010). Engagementförderung als Demokratiepolitik. Besichtigung einer Reformbaustelle. In T. Olk, A. Klein \& B. Hartnuß (Hrsg.), Engagementpolitik. Die Entwicklung der Zivilgesellschaft als politische Aufgabe (S. 611-636). Wiesbaden: VS.

Schütze, F. (1984). Kognitive Figuren des autobiographischen Stehgreiferzählens. In M. Kohli \& R. Günther (Hrsg.), Biographie und soziale Wirklichkeit. Neue Beiträge und Forschungsperspektiven (S. 78-117). Stuttgart: Metzler.

Simonson, J., Vogel, C., \& Tesch-Römer, C. (2017). Freiwilliges Engagement in Deutschland. Der Deutsche Freiwilligensurvey 2014. Wiesbaden: Springer VS.

Snyder, M., \& Omoto, A.M. (2008). Volunteerism: social issues perspectives and social policy implications. Social Issues and Policy Review, 2(1), 1-36.

Straub, J. (2004). Identität. In F. Jaeger \& B. Liebsch (Hrsg.), Handbuch der Kulturwissenschaften. Grundlagen und Schlüsselbegriffe (Bd. 1) (S. 277-303). Stuttgart: Metzler.

Varga, V., \& Munsch, C. (2014). Kontextspezifische Positionierungen: Darstellung eines Forschungszugangs und einer Analysestrategie am Fallbeispiel einer Lehrkraft „,mit Migrationshintergrund“. Forum: Qualitative Sozialforschung, 15(3). http://nbn-resolving.de/urn:nbn:de:0114-fqs140330. [Letzter Zugriff am 11. September 2017]

Weber, M. (2014). Wirtschaft und Gesellschaft. Soziologie. Tübingen: Mohr Siebeck.

Wilson, J. (2012). Volunteerism research. A review essay. Nonprofit and Voluntary Sector Quarterly, 41(2), 176-212. https://doi.org/10.1177/0899764011434558. [Letzter Zugriff am 19. Juni 2018]. 